\title{
Recent progress on the research of high strength hydrogels
}

\author{
Da Zhang ${ }^{1,2 a^{*}}$, Liang Chen ${ }^{1,2,3 \mathrm{~b}}$, Zaiqin Wang ${ }^{1,2,3 \mathrm{c}}$ and Tao Wei ${ }^{1,2 \mathrm{~d}}$ \\ ${ }^{1}$ Changjiang River Scientific Research Institute, Wuhan, 430010, China \\ ${ }^{2}$ Collaborative Innovation Center for Geo-hazards and Eco-Environment in Three Gorges Area, \\ Yichang, 443002, China \\ ${ }^{3}$ National Dam Safety Research Center, Wuhan, 430010, China

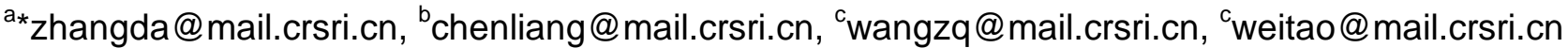

Keywords: hydrogel, high strength, mechanical property.

Abstract. The hydrogel is a three-dimensional reticular polymer with high water content, which was used in various fields such as medicine, biology and environmental protection. However, the characteristic of lower mechanical properties limited its application. Therefore, the improvement of the mechanical strength of hydrogel has become the focus of researchers. This paper mainly introduced the progress of research on synthesis of several kinds of high strength hydrogel, including slip ring hydrogel, Tetra-PEG hydrogel, internal passing network hydrogel, double network hydrogel and nanocomposite hydrogel. The analysis of the influence factors on mechanical properties was discussed.

\section{Introduction}

The hydrogel is a kind of soft wet material, consisting of cross-linking macromolecules and water. The appearance of hydrogel is solid, but it has liquid micro composition. Small molecules have high permeability to pass through. The changes of exudation or absorption of water are reversible, which can be produced in response to various stimuli, such as light, temperature, $\mathrm{pH}$, ionic strength and chemical reaction. In the past twenty years, the sensor volume change and the driving properties of hydrogel have been extensively studied. Recently, the hydrogel has attracted a lot of interest in biological materials because of its similar property to soft tissue. For example, it was applied to the extracellular matrix, artificial organs and biological model of human body tissue [1].

However, the traditional hydrogel composed of a hydrophilic polymer is weak, and easy to break. The tensile stress is usually less than $1 \mathrm{MPa}$, and the strain is less than $100 \%$. But the strength of actual biological tissue is much greater. The compression fracture stress of cartilage is $36 \mathrm{MPa}$, which contains $75 \mathrm{wt} \%$ of water. At the same time, traditional hydrogels also exhibited very low resistance to extension crack. The poor mechanical characteristics of hydrogel hindered the wide application of this kind of materials [2]. So far, most of the hydrogel can only be used in the areas where mechanical properties are not required, such as drug delivery device and water absorption device.

The reason for the poor mechanical strength of the hydrogel is the nature of its property similar to solution. The density of the polymer chains is low, and the friction force between the chains is small. Another reason is that the hydrogel network structure formed in the process of gelation has inhomogeneity. When the force is applied to the inhomogeneous structure, stress will be concentrated in the shortest chain, and a very small force can make the gel breaking. Therefore, scientists have been devoted to synthesizing homogeneous network structure, such as slip ring (SR) hydrogel, 4-polyethylene glycol hydrogel, double network (DN) hydrogel, nano composite (NC) hydrogel, etc.

\section{Slip ring hydrogel}

Slip ring hydrogel also was called topological structure hydrogel. The typical structure is characterized with cross-linked ring type [3]. It was synthesized by linear molecular chain of PEG crossing through the $\alpha$-cyclodextrin $(\alpha-C D)$ ring, with bulky groups sealing the PEG chain, and then the $\alpha-C D$ was chemically crosslinked to form hydrogel. The structure diagram is shown in Fig. 1 [4]. The crosslinking points of figure 8 can slide in the PEG chain, to play the role of pulley. This kind of dynamic covalent chemistry of pulley crosslink point is the essential characteristic of the topological structure, which is different from other traditional chemical crosslinked hydrogels. When the gel is 
subjected to tension, the cross link can be relatively sliding, so that the external force can be uniformly dispersed in the polymer chain, which makes the hydrogel has a high tensile strength and elongation.

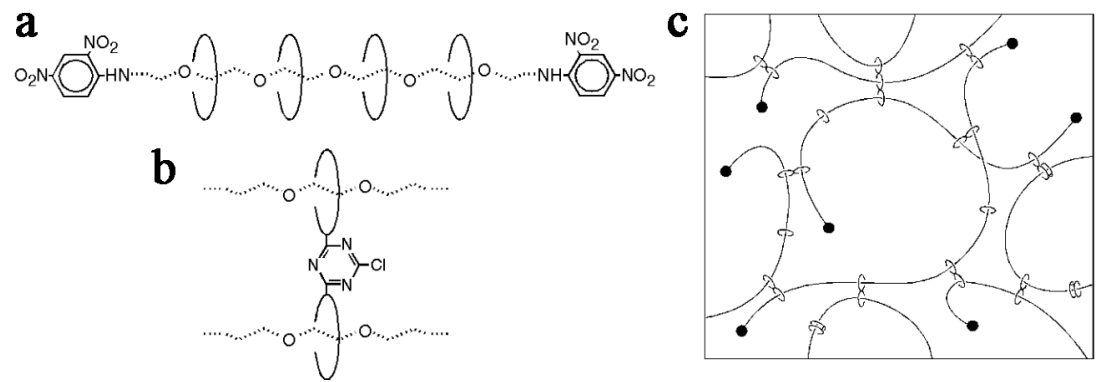

Fig. 1 Schematic diagram of the SP hydrogel prepared by the sparse polyrotaxane crosslinked covalently with cyclodextrin

\section{Tetra-PEG hydrogel}

The uneven structure of the hydrogel will affect the mechanical strength. Researchers have been searching for uniform construction of hydrogel. Sakai prepared a Tetra-PEG hydrogel using a large molecule with a tetrahedral structure, as shown in Fig. 2 [5]. Two kinds of large molecules with tetrahedral structure are alternately connected, avoiding the occurrence of self-reaction. The A-B reaction type is simple, and consistent with the classic Flory theory [6]. When the two kinds of polymer solution are mixed, the cross-linking between amino and succinimide ester occurred with the regulation of $\mathrm{pH}$ value.

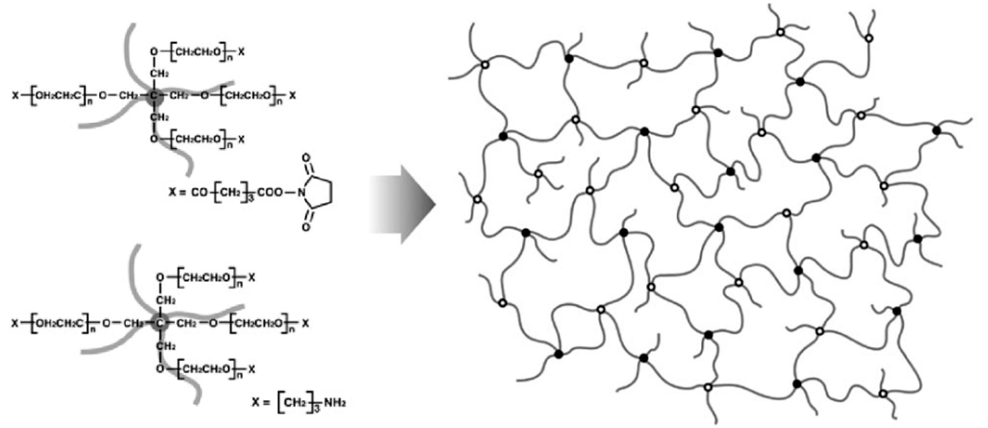

Fig. 2 Scheme of fabrication process of Tetra-PEG network.

Tetra-PEG hydrogel is different from the traditional internal polymer hydrogel, which has swing and entangled chains. Its interior is almost ideal network, and the dynamic light scattering results showed that tetrahedral macromolecules are alternately connected to form a network structure with length of two arms. The mesh size in the hydrogel structure is uniform. The relationship of stress and strain can be described by the ideal network formula, and it is one of the most regular hydrogels.

\section{Internal Passing Network hydrogel}

Internal Passing Network (IPN) hydrogel is formed by two kinds of polymer interpenetrating and winding by each other [7]. The two kinds of polymer have different function, so as to realize the complementary properties of different components. Because of the special characteristics of IPN hydrogel, such as interface interpenetrating and dual phase, it has a synergistic effect on mechanical strength. IPN hydrogel with thermo sensitive properties and good swelling property has been widely used in drug delivery. Lee et al [8] obtained the IPN hydrogels from the PEG monomer, chitosan and glutaraldehyde as crosslinking agent under ultraviolet light. The equilibrium water content (EWC) is in the range of $74-97 \%$. The crystallinity of PEGM in IPN network is significantly lower than that of PEGM. Two glass transition temperatures (Tg) were found in the inter network structure, which indicated the existence of phase separation in IPN hydrogel. 


\section{Double network hydrogel}

Double network (DN) hydrogel is a new type of high strength hydrogels developed in IPN hydrogel [9,10]. It was firstly reported by Gong et al in 2004, as shown in Fig. 3. DN gel is a special network structure which is composed of two kinds of non-symmetrical polymer. The minor component is highly crosslinked by polyelectrolyte (rigid frame), and the main component is low crosslinked by neutral polymer (ductile material), in which the former and the latter are called the first network and second network. The synthetic process must be in accordance with the order to achieve high mechanical strength. In appropriate conditions, they have good hardness (elastic modulus of 0.1-1.0 MPa), strength (tensile stress of 1-10 MPa, tensile strain of 1000-2000\%; compression failure stress of 20-60 $\mathrm{MPa}$, compression strain of 90-95\%), and toughness (tear fracture energy of $100-1000 \mathrm{Jm}^{-2}$ ). These excellent mechanical properties can be comparable with rubber.
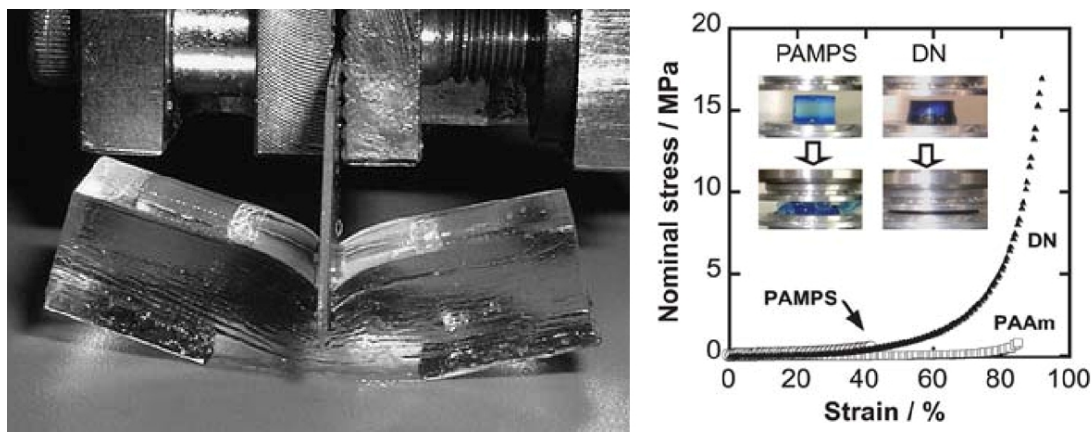

Fig. 3 The photography of tough DN hydrogel and the their comprehensive curves

\section{Nanocomposite hydrogel}

The nanocomposite hydrogel was obtained by in situ polymerization, using inorganic clay nanosheets as crosslinking points. The network structure was formed by the non-covalent force of electrostatic interaction. So the internal structure is uniform, avoiding the crosslinking density of the uneven in the reaction of organic crosslinking agent $[11,12]$. This system was originated by Japanese professor Haraguchi, using NIPAm as the organic monomer, clay nanosheets as crosslinking points, potassium persulfate (KPS) as initiator and N, N, N, N- tetra methyl ethylenediamine (TEMED) as accelerator. The nanocomposite hydrogel has excellent mechanical properties. When the water content is $90 \%$, the elongation of the hydrogel can reach $1000 \%$ or more. Moreover, the NC hydrogel also has the characteristics of high transparency, high swelling performance and rapid response to temperature changes, which are difficult for other hydrogel containing these features at the same time. The network structure model of NC hydrogel is shown in Fig. 4.
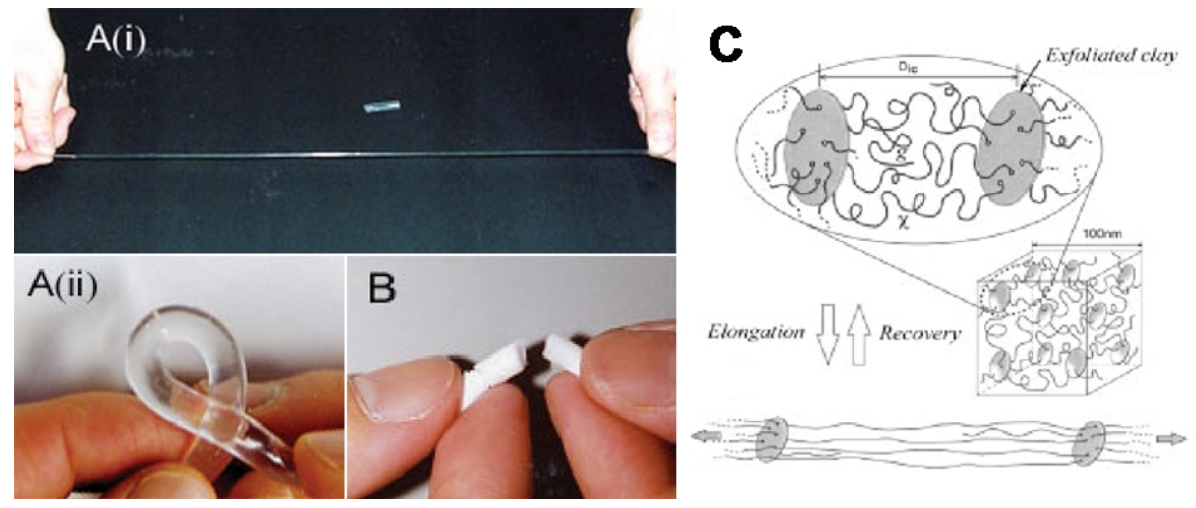

Fig. 4 The images of nanocomposite hydrogels (A), OR gels (B) and the internal structure of NC hydrogels $(\mathrm{C})$ 


\section{Summary}

The study of the hydrogel is related to many subjects, such as medical, biological engineering, polymer science, life science, and has become a hot academic field. In order to further expand the application range, researchers use a variety of methods to improve the mechanical strength of the hydrogel. Hydrogels with excellent mechanical properties have a broad application prospect in the field of biological materials, as used for succedaneum of particularly artificial cartilage, artificial muscles, tendons and ligaments, artificial cornea, etc.

\section{Acknowledgments}

This work was supported by Ministry of Water Resources nonprofit industry research project (Grant No. 201301023), National Natural Science Foundation of China (Grant No. 51378078), Thanks for the supporting by Innovation Team (CKSF2012051/CL) and International Science \& Technology Cooperation Program of China (Grant No. 2010DFB70470).

\section{References}

[1] Y. Tanaka, J. P. Gong, Y. Osada, Novel hydrogels with excellent mechanical performance, Prog. Polym. Sci. 30(2005) 1-9.

[2] J. P. Gong, Why are double network hydrogels so tough? Soft Matter 6(2010) 2583-2590.

[3] Y. Okumura, K. Ito, The polyrotaxane gel: A topological gel by figure of eight crosslinks, Adv. Mater. 13(2001) 485-487.

[4] C. Zhao, Y. Domon, Y. Okumura, Sliding mode of cyclodextrin in polyrotaxane and slide-ring gel, J Phys. Condens. Matter 17(2005) S2841-S2846.

[5] T. Sakai, T. Matsunaga, Y. Yamamoto, Design and fabrication of a high-strength hydrogel with ideally homogeneous network structure from tetrahedron-like macromonomers, Macromolecules 41(2008) 5379-5384.

[6] K. Nishi, K. Fujii, M. Chijiishi, Kinetic Study for AB-Type Coupling Reaction of Tetra-Arm Polymers, Macromolecules 45(2011) 1031-1036.

[7] S. J. Kim, S. J. Park, S. I. Kim, Swelling behavior of interpenetrating polymer network hydrogels composed of poly (vinyl alcohol) and chitosan, Reactive and Functional Polymers 55(2003) 53-59.

[8] S. J. Lee, S. S. Kim, Y. M. Lee, Interpenetrating polymer network hydrogels based on poly (ethylene glycol) macromer and chitosan, Carbohydrate Polymers 41(2000) 197-205.

[9] J. P. Gong, Y. Katsuyama, T. Kurokawa, Double-Network Hydrogels with Extremely High Mechanical Strength, Adv. Mater. 15(2003) 1155-1158.

[10] A. Nakayama, A. Kakugo, J. P. Gong, High mechanical strength double-network hydrogel with bacterial cellulose, Adv. Funct. Mater. 14(2004) 1124-1128.

[11] K. Haraguchi, T. Takehisa, Nanocomposite hydrogels: a unique organic-inorganic network structure with extraordinary mechanical, optical, and swelling/de-swelling properties, Adv. Mater. 14(2002) 1120-1124.

[12] K. Haraguchi, H. J. Li, Control of the Coil to Globule Transition and Ultrahigh Mechanical Properties of PNIPA in Nanocomposite Hydrogels, Angew. Chem. Int. Ed. 44(2005) 6500-6504. 